\title{
Effects of a Multidisciplinary Care Program on Disability, Autonomy, and Nursing Needs in Subjects Recovering From Acute Respiratory Failure in a Chronic Ventilator Facility
}

\author{
Michele Vitacca MD, Mara Paneroni PT, Roberta Peroni PT, Luca Barbano MD, \\ Valmira Dodaj PT, Giancarlo Piaggi PT, Fabio Vanoglio PT, Alberto Luisa MD, \\ Amerigo Giordano MD, and Piero Ceriana MD
}

\begin{abstract}
BACKGROUND: The aim of this study was to analyze the effects of a multidisciplinary program carried out in a chronic ventilator facility on disability, autonomy, and nursing needs of patients after a prolonged ICU stay. Secondary outcome measures were survival, weaning rate, chronic ventilator facility stay, and discharge destination. METHODS: Multidisciplinary assessment, clinical stabilization, weaning attempts, and a new Disabled Patients Autonomy Planning tool to assess daily care needs were investigated in $\mathbf{2 4 0}$ subjects in a chronic ventilator facility (52 subjects after cardiovascular surgery, 60 subjects with acute respiratory failure, 71 subjects with COPD, and 57 subjects with neurological disease). RESULTS: At admission, nursing needs, disability, and autonomy differed according to diagnosis $(P<.001)$; weaned subjects had greater nursing needs $(P<.001)$ and disability $(P=.0014)$ than unweaned subjects. During the stay, $13.8 \%$ of the subjects died irrespective of diagnosis $(P=.12) ; 47 \%(P<.001)$ were weaned with significant differences $(P<.007)$ by diagnosis. In the 207 surviving subjects, nursing needs increased as disability increased $(r=0.59$, $P<.001)$ and autonomy decreased $(\mathrm{r}=-0.66, P<.001)$; disability and autonomy were interrelated $(\mathrm{r}=0.61, P<.001)$. Oxygen saturation, hypercapnia, dyspnea, disability, autonomy, and nursing needs significantly improved (all, $P<.001$ ). Fifty-nine percent of the subjects were discharged home. Subjects discharged to nursing homes presented mainly neurological diseases, being more disabled and less autonomous, with higher nursing needs (all, $P<.04$ ). Mechanical ventilation use and tracheostomy increased the probability of being discharged to a nursing home (odds ratio $[\mathrm{OR}]$ of $1.84, P=.04$; OR $2.47, P=.003$, respectively). Mortality was higher in subjects who were ventilated (OR 8.44, $P<.001)$, male $(O R 2.64, P=.01)$, elderly $(P<.001)$, or malnourished $(P=.01)$ and in subjects with low autonomy $(P<.001)$, greater nursing needs $(P=.002)$, and more severe disabilities $(P=.04)$. CONCLUSIONS: A specialized tailored multidisciplinary program in subjects after an ICU stay contributed to recovery from disability, autonomy, and fewer nursing needs irrespective of diagnosis. Subjects discharged to a nursing home were the most severely disabled. Key words: prolonged mechanical ventilation; weaning; chronic care facilities; COPD; postsurgical; long-term hospital. [Respir Care 2014;59(12):1863-1871. (C) 2014 Daedalus Enterprises]
\end{abstract}

\section{Introduction}

In the last $15 \mathrm{y}$, with the increased availability of beds in ICUs and improved levels of care, a category of patients

Dr Vitacca and Barbano, Ms Paneroni, and Ms Dodaj are affiliated with the Divisione di Pneumologia Riabilitativa, Ms Peroni and Dr Giordano are affiliated with the Divisione di Cardiologia Riabilitativa, and $\mathrm{Mr}$ labeled as survivors of a catastrophic illness has emerged. ${ }^{1}$ These patients commonly suffer from a wide range of

\footnotetext{
Vanoglio and Dr Luisa are affiliated with the Divisione di Neurologia Riabilitativa, Fondazione Salvatore Maugeri, Istituto di Ricovero e Cura a Carattere Scientifico, Lumezzane (Brescia), Italy. Mr Piaggi and Dr Ceriana are affiliated with the Divisione di Pneumologia Riabilitativa, Fondazione Salvatore Maugeri, Istituto di Ricovero e Cura a Carattere Scientifico, Pavia, Italy.
} 


\section{Multidisciplinary Care in Acute Respiratory Failure}

physical disabilities that may be further aggravated by muscle wasting, neuromyopathies, and poor nutritional status. ${ }^{2-4}$ Hospitalization and recovery can be very prolonged in this subset of patients. ${ }^{1}$ Indeed, this condition may increase risk of early mortality 5,6 and late pulmonary complications, ${ }^{7}$ involving increased health costs. ${ }^{8}$ Patients needing prolonged mechanical ventilation (PMV) ${ }^{9}$ may require transfer to a protected environment such as a chronic ventilator facility, ${ }^{3,10}$ which studies have shown can offer some clinical and financial advantages over a prolonged ICU stay. $3,11,12$ In particular, patients may receive a prompt and dedicated physiotherapy rehabilitation program in chronic ventilator facilities, ${ }^{1,3,13}$ and transfer from an ICU to a chronic ventilator facility substantially improved patients' ambulation and activities of daily life irrespective of the underlying pathophysiology, avoiding unnecessary immobilization $^{14}$

To date, the role of comprehensive rehabilitation in critically ill adults still remains controversial ${ }^{13}$ or not fully established ${ }^{15}$ : patients who have survived a recent acute respiratory failure (ARF) event can benefit from an individually tailored physiotherapy rehabilitation program that improves clinical indices, respiratory and peripheral muscle strength, activities of daily life, exercise tolerance, and disability scores. ${ }^{14,16-24}$

Once the precipitating cause of the ARF episode has been solved, these chronically ill patients still require care due to their huge motor disabilities, clinical dependence (oxygen, mechanical ventilation, nutritional, and communication device needs), and nursing necessities, ${ }^{3}$ which the care team (physiotherapist, doctor, and nurse) must measure to better guide the daily care of the patient. No study has been performed with a large patient sample to investigate patient needs with these 3 different perspectives combined.

In this observational cohort study, we investigated a selected population of severely disabled subjects, with motor disabilities, surviving a recent ICU stay and admitted to a chronic ventilator facility within a rehabilitation center. Our primary objective was to analyze whether comprehen-

Supplementary material related to this paper is available at http:// www.rcjournal.com.

The study was partially supported by Independent Research Grant 2010 (Decreto 5685_Maggio 2010, identificativo atto 475) from Regione Lombardia, Italy. The authors have disclosed no conflicts of interest.

Correspondence: Michele Vitacca MD, Divisione di Pneumologia Riabilitativa, Fondazione Salvatore Maugeri, Istituto di Ricovero e Cura a Carattere Scientifico, Via Giuseppe Mazzini 129, 25065 Lumezzane (Brescia), Italy. E-mail: michele.vitacca@fsm.it.

DOI: $10.4187 /$ respcare. 03030

\section{QUICK LOOK}

\section{Current knowledge}

Patients needing prolonged mechanical ventilation may require transfer to a chronic ventilator facility, which can offer some clinical and financial advantages over a prolonged ICU stay. The role of comprehensive rehabilitation in critically ill adults is this environment remains controversial or not fully established.

\section{What this paper contributes to our knowledge}

In subjects discharged from the ICU, irrespective of their diagnosis, a multidisciplinary care program contributed to improved disability, autonomy, and nursing needs, with these indices being inter-related. Mortality and weaning are related to clinical and disability status and to their reciprocal improvement over time. Patients discharged to a nursing home are the most severely disabled.

sive autonomy and nursing needs can be modified by a structured specialized multidisciplinary in-patient intervention. Secondary outcome measures were survival, weaning success, chronic ventilator facility stay, and discharge destination.

\section{Methods}

All study procedures were performed in conformity with the Declaration of Helsinki. The Salvatore Maugeri Foundation ethics committee approved the study (751 CEC 21/7/2011). Informed written consent for the study procedures was obtained from all subjects and/or their relatives.

\section{Subjects}

This prospective cohort study was carried out on eligible subjects consecutively admitted to the Salvatore Maugeri Foundation in Lumezzane (Brescia) and Pavia, Italy, for a comprehensive rehabilitation intervention between March 15, 2011, and March 15, 2013 (Fig. 1).

Eligibility Criteria. To be eligible for the study, subjects had to be $>18$ y old, with a clinical history of recent (within the last 3 months) severe ARF, a period of mechanical ventilation in the ICU lasting for at least 20 consecutive days, severe disability, and loss of autonomy in daily life but not nearing end-of-life. Resolution of the clinical conditions underlying the onset of ARF and clinical stability were defined as: arterial pressure of $<20 \%$ of daily variability (absence of hypotension or 


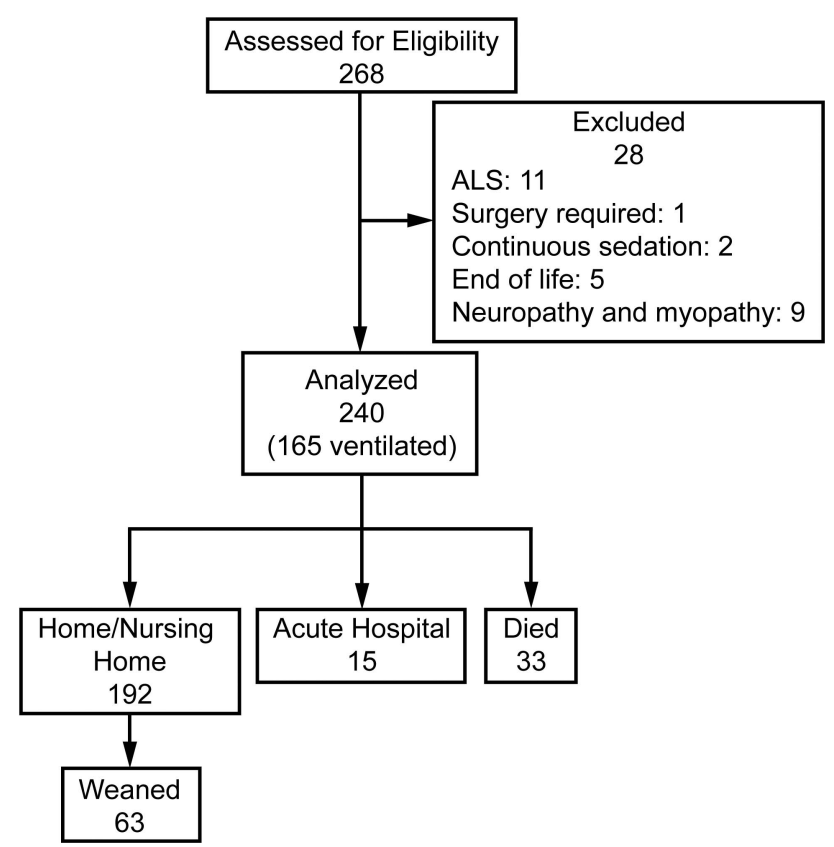

Fig. 1. Flow chart of study design showing subject recruitment and outcome. ALS = amyotrophic lateral sclerosis.

hypertension and vasopressors needs), no severe arrhythmias, $\left.\mathrm{P}_{\mathrm{aO}_{2}}\right) / \mathrm{F}_{\mathrm{IO}_{2}}>250$, stabilized breathing patterns (breathing frequency of $<30$ breaths/min on mechanical ventilation or breathing spontaneously), hemoglobin of $>7 \mathrm{~g} / \mathrm{dL}$, temperature of $<38^{\circ} \mathrm{C}$, normal platelet and white blood cell counts, no contraindications to exercise due to neurological or orthopedic diseases, absence of urgent surgical indications during current hospitalization, and no embolism within the last $30 \mathrm{~d}$.

Exclusion Criteria. Patients with amyotrophic lateral sclerosis, patients who required immediate surgery, continuous sedation, hemodialysis, or peritoneal dialysis, patients with a diagnosis of ICU-acquired neuropathy and myopathy demonstrated via electromyogram, and patients who refused to participate were excluded.

\section{Program}

The rehabilitation program, which was based on a multidisciplinary team of doctors, nurses, and respiratory physiotherapists, consisted of:

(1) Medical Treatment. The intervention by doctors was aimed at addressing the root causes, complications, and acquired comorbidities leading to the subject's previous ICU admission.

(2) Weaning from Mechanical Ventilation. For mechanically ventilated subjects, the weaning program was conducted by doctors after evaluating a subject's clinical stability and spontaneous breathing trial results. ${ }^{25}$ If the subject failed the spontaneous breathing trial, weaning was done gradually by progressively reducing the inspiratory support or increasing the time of spontaneous breathing. During this process, the subject was carefully observed to detect any clinical deterioration. Subjects were considered weaned after at least $48 \mathrm{~h}$ of respiratory autonomy and successfully weaned once they were able to sustain spontaneous breathing for at least 7 consecutive days. ${ }^{25}$

(3) Intensive Nursing. A nurse was involved in all activities of patient care, including administration of prescribed drug therapies, constant checks, cleaning, sore prevention, and wound or suction management.

(4) Individualized and Integrated Respiratory Physiotherapy. A dedicated respiratory physiotherapist was assigned to help the subject achieve the maximum possible autonomy for long-term maintenance of the acquired results. The respiratory physiotherapy program was individually tailored based on the initial structured evaluation of the subject. The intervention was focused on exercises of passive and active assisted mobilization, maintenance of body posture, electrical stimulation of leg muscles, sitting and standing postural control exercises, arm and leg strength/endurance training on a cycle ergometer or treadmill, walking assistance, lung expansion exercises, and bronchial hygiene. The time period of the rehabilitation intervention was $1 \mathrm{~h} / \mathrm{d}$ and could be performed in a single daily session or in two 30-min sessions (one in the morning and one in the afternoon) $6 \mathrm{~d}$ /week (excluding Sundays). However, on Sundays, mobilization of the subject in the chair was performed by nurses. The exercise training program was conducted independently of the weaning process. During the weaning period, the rehabilitation sessions were conducted using the mode of therapy deemed most comfortable by the subject (eg, mechanical ventilation, if still receiving, or with a level of oxygen sufficient to produce $\mathrm{O}_{2}$ saturation of $>90 \%$ during exercise). Respiratory assistance was gradually reduced over time, and the respiratory physiotherapist constantly monitored the subject for signs of cardiorespiratory distress during the sessions.

\section{Measurements}

At time of admission to the units, subject demographic data, admission diagnosis (post-cardiovascular surgery, ARF, COPD, or neurological diseases), length of mechanical ventilation, ICU stay, pre-existing comorbidities via the Cumulative Illness Rating Scale, ${ }^{26}$ and dyspnea by means of the Borg scale 27 were collected. Arterial blood gas analysis was performed, and albumin levels, presence 


\section{Multidisciplinary Care in Acute Respiratory Failure}

of tracheostomy and mechanical ventilation, Malnutrition Universal Screening Tool ${ }^{28}$ score to identify whether the subject was malnourished or at risk of malnutrition or obesity (best $=0$, worse $=2$ ), and the Acute Physiology and Chronic Health Evaluation II score ${ }^{29}$ were determined.

At time of admission and discharge from the units, the level of disability using the Barthel Index (score range of $0-20$, with low scores indicating severe disability), ${ }^{30}$ nursing dependence using the Gussago Nursing Scale (GNS; score range of $0-44$, with high scores indicating high dependence), ${ }^{31}$ and the Disabled Patients Autonomy Planning (DPAP) score were determined. The DPAP tool is a triage procedure used to make an instant evaluation of a patient's global autonomy. It was developed ad hoc by our group as a guide for health workers in the daily care of patients from the different perspectives of the personnel involved in these units (medical doctors, physiotherapists, and nurses). First, a panel of experts discussed the contents of autonomy and identified available tools that could be modified and adapted for a rehabilitation setting to suit our subjects' characteristics. Second, the experts judged each item as to whether it was appropriate, related to the general context, and important. The final tool consists of 24 items that score the level of neurological, respiratory, and motor dependence of a patient in basic activities of daily life, infusion drug support, and home management availability. Details of the items and scoring system based on dichotomous (yes/no) responses are available in Appendix 1 (score range of 1-24, with higher scores reflecting greater autonomy). (See the supplementary materials at http://www.rcjournal.com.) Before its use in this study, the DPAP tool was tested for clarity of the items in a pilot group of 40 subjects. As a result, 2 changes were made to improve feasibility and understanding. To verify its reliability, the DPAP tool was retested 3 times on the same day in 16 subjects, changing the profile of health personnel who administered the tool (doctor, physiotherapist, and nurse). The first and third tests were compared. The intraclass correlation coefficient was 0.85 .

At discharge from the units, we also evaluated weaning success, mortality rate, stay, and discharge destination.

\section{Statistical Analysis}

Analyses were carried out using the statistical software package Stata 11.2 (StataCorp, College Station, Texas). Descriptive analysis was conducted using mean \pm SD for continuous variables and percentage for dichotomous variables. Risk of death, weaning success, and discharge to a nursing home were predicted using the odds ratio (OR) for dichotomous variables and the unpaired Student $t$ test for continuous variables. Mortality, weaning, and home discharge frequency by diagnosis were analyzed using the Pearson chi-square test. Changes in clinical variables and
Table 1. Characteristics of the Study Population

\begin{tabular}{lc}
\hline \hline & Values \\
\hline Enrolled subjects, $N$ & 240 \\
Age (mean \pm SD), y & $68 \pm 13$ \\
Males, $n(\%)$ & $128(53.3)$ \\
Admission diagnosis & \\
$\quad$ Post-surgical, $n(\%)$ & $52(21.6)$ \\
$\quad$ ARF, $n(\%)$ & $60(25)$ \\
COPD, $n(\%)$ & $71(29.5)$ \\
$\quad$ Neurological diseases, $n(\%)$ & $57(23.7)$ \\
ICU ventilator stay (mean \pm SD), d & $29 \pm 7$ \\
CIRS 1 (mean \pm SD) & $1.88 \pm 0.44$ \\
CIRS 2 (mean \pm SD) & $4.49 \pm 1.46$ \\
Albumin (g/dL) & $3.01 \pm 0.48$ \\
$\mathrm{P}_{\text {aO }} / F_{\text {IO }}$ (mean \pm SD) & $258 \pm 82$ \\
$\mathrm{P}_{\text {aCO }}$ (mean \pm SD), mm Hg & $52 \pm 11$ \\
pH (mean \pm SD) & $7.44 \pm 0.07$ \\
MUST score (mean \pm SD) & $1.91 \pm 0.90$ \\
APACHE II score (mean \pm SD) & $12 \pm 6$ \\
Borg dyspnea score (mean \pm SD) & $5.48 \pm 3.06$ \\
& \\
\hline ARF $=$ acute respiratory failure & \\
CIRS $=$ Cumulative Illness Rating Scale & \\
APACHE II $=$ Acute Physiology and Chronic Health Evaluation II & \\
MUST = Malnutrition Universal Screening Tool & \\
\hline
\end{tabular}

disability, autonomy, and nursing needs during hospitalization were investigated by the paired $t$ test. At time of admission to the units (t0), the univariate Pearson correlation was performed between the scales of disability, autonomy, and nursing needs. Analysis of variation was performed to verify differences in disability, autonomy, and nursing need scores at admission (t0) and discharge (t1) and to compare changes between $\mathrm{t} 1$ and t0 among the different diagnoses. If the Fisher exact test was significant, a post hoc analysis with the Bonferroni correction was performed to test the statistical differences between variables. For all analyses, $P<.05$ was considered as statistically significant. A Kaplan-Meier curve was performed to describe cumulative subject survival after admission. A log-rank test was performed to find survival function differences among diagnoses.

\section{Results}

A flow chart showing the design of the study is presented in Figure 1. Subjects received only episodic mobilization interventions in the ICUs from which they were referred.

Table 1 shows the characteristics of the study population: 52 subjects recovering from cardiovascular surgery, 60 subjects with severe ARF (pneumonia, 45\%; sepsis, $30 \%$; trauma, 15\%; and ARDS, 10\%), 71 subjects with COPD, and 57 subjects with neurological diagnoses (stroke, 
Table 2. Changes in Disability, Autonomy, and Nursing Needs in 207 Surviving Subjects

\begin{tabular}{|c|c|c|c|c|c|c|}
\hline & Total & Post-Surgical & ARF & COPD & $\begin{array}{l}\text { Neurologic } \\
\text { Diseases }\end{array}$ & $\begin{array}{c}P \\
\text { (ANOVA) }\end{array}$ \\
\hline Subjects, $n$ & 207 & 37 & 54 & 63 & 53 & \\
\hline \multicolumn{7}{|l|}{ Barthel index } \\
\hline to & $4.56 \pm 4.34$ & $4.63 \pm 4.17$ & $4.93 \pm 3.96$ & $6.41 \pm 4.57$ & $1.81 \pm 3.13^{* \dagger}$ & $<.001$ \\
\hline $\mathrm{t} 1$ & $9.67 \pm 5.45 \ddagger$ & $10.29 \pm 5.28 \ddagger$ & $10.60 \pm 4.66 \ddagger$ & $11.78 \pm 4.25 \ddagger$ & $5.49 \pm 5.60 \ddagger \S$ & $<.001$ \\
\hline$\Delta$ & $4.82 \pm 4.37$ & $5.50 \pm 5.27$ & $5.23 \pm 3.30$ & $5.08 \pm 3.88$ & $3.49 \pm 4.87$ & .10 \\
\hline \multicolumn{7}{|l|}{ DPAP } \\
\hline to & $8.73 \pm 3.73$ & $8.45 \pm 3.72$ & $9.25 \pm 3.59$ & $10.21 \pm 3.51 \|$ & $6.54 \pm 3.14 \uparrow \llbracket$ & $<.001$ \\
\hline $\mathrm{t} 1$ & $16.53 \pm 4.74 \ddagger$ & $15.81 \pm 5.49 \ddagger$ & $21.06 \pm 8.43 \ddagger$ & $17.45 \pm 4.16 \ddagger$ & $11.60 \pm 5.49 \ddagger * *$ & .01 \\
\hline$\Delta$ & $7.42 \pm 14.12$ & $6.74 \pm 5.19$ & $11.29 \pm 27.60$ & $7.05 \pm 4.23$ & $4.76 \pm 5.18$ & .14 \\
\hline \multicolumn{7}{|l|}{ GNS } \\
\hline to & $24.25 \pm 6.67$ & $24.39 \pm 6.94$ & $24.38 \pm 6.06$ & $22.27 \pm 7.04$ & $26.53 \pm 5.90 \dagger \dagger$ & .005 \\
\hline $\mathrm{t} 1$ & $13.54 \pm 7.78 \ddagger$ & $12.55 \pm 7.84 \ddagger$ & $11.58 \pm 6.96 \ddagger$ & $11.44 \pm 6.32 \ddagger$ & $18.94 \pm 7.84 \ddagger \S$ & $<.001$ \\
\hline$\Delta$ & $-10.12 \pm 7.69$ & $-11.07 \pm 8.64$ & $-12.06 \pm 7.11$ & $-10.47 \pm 7.00$ & $-7.02 \pm 7.51$ 中 & .007 \\
\hline 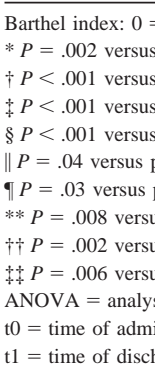 & $\begin{array}{l}20=\text { best. Disabled Pat } \\
\text { Irgical subjects. } \\
\text { s with acute respiratory } \\
\text { Irgical subjects and subj } \\
\text { gical subjects. } \\
\text { gical subjects. } \\
\text { cts with ARF. } \\
\text { cts with COPD. } \\
\text { ts with ARF. } \\
\text { ariance } \\
\text { unit }\end{array}$ & $\begin{array}{l}\text { utonomy Planning (DPA } \\
\text { (ARF) and COPD. } \\
\text { h ARF and COPD. }\end{array}$ & 1: $0=$ worst, $24=$ best & Nursing Scale (GNS): & st, 44 = worst. & \\
\hline
\end{tabular}

48\%; post-cardiac arrest, 18\%; Guillain-Barré syndrome, $14 \%$; no degenerative neuromuscular diseases, $13 \%$; Parkinson disease, 3.5\%; and astrocytoma, 3.5\%). Diagnosis was equally distributed. Subjects presented with several comorbidities and dyspnea and were at risk of malnutrition; they also had high disability, low autonomy, and serious nursing needs. During their stay, 33 (13.8\%) subjects died irrespective of diagnosis $(P=.12)$.

At admission, 183 subjects were tracheostomized $(76.2 \%)$, and 165 of these subjects were ventilated $(90.16 \%)$. Subjects stayed a mean time of $40 \mathrm{~d}$ in the ICU, with an average of $44 \pm 43$ additional days in the rehabilitation unit (range of 7-180 d).

Table 2 shows data at admission (t0) and discharge (t1) and changes during hospital stay $(\Delta)$ in disability, autonomy, and nursing needs in the 207 surviving subjects according to diagnosis at entry. At discharge, disability, autonomy, and nursing needs significantly improved $(P<.001)$ for all subjects irrespective of diagnosis. At both admission and discharge, disability, autonomy, and nursing needs differed significantly among the diagnoses. Subjects with neurological disease showed the worst level of disability, autonomy, and nursing needs at both admission and discharge. The neurological disease group also showed less improvement in nursing needs compared with the ARF group.
Figure 2 shows the relationship among the Barthel, DPAP, and GNS scores at admission. At admission, disability and nursing needs were significantly related, that is, nursing needs increased as disability increased $(\mathrm{r}=0.59$, $P<.001$ ), confirming that subjects with higher disability also required higher nursing needs. Nevertheless, as Fig. $2 \mathrm{C}$ shows, some subjects with high disability (Barthel score of 0-5) did not have high nursing needs (GNS score of $<20)$. At admission, autonomy and nursing needs $(\mathrm{r}=0.66$, $P<.001)$ were significantly inversely related, confirming that subjects with less autonomy also required higher nursing needs (Fig. 2A). In addition, some subjects with low autonomy (DPAP score of 0-10) did not have high nursing needs (GNS score of $<20$ ). Disability and autonomy were also significantly related $(\mathrm{r}=0.61, P<.001)$ (Fig. 2B). Some subjects who were not dependent according to the DPAP tool (score of $>10$ ) were disabled according to the Barthel index.

Figure 3 shows the cumulative subject survival after admission. Thirty-three of 240 (13.75\%) subjects died following admission. No significant difference was found in survival rate among diagnoses $(P=.12)$. Median time to death was $23 \mathrm{~d}$ (range of 5-187). No significant differences were found in median times between the different diseases, but ventilated subjects had an $8.44(P<.001)$ 

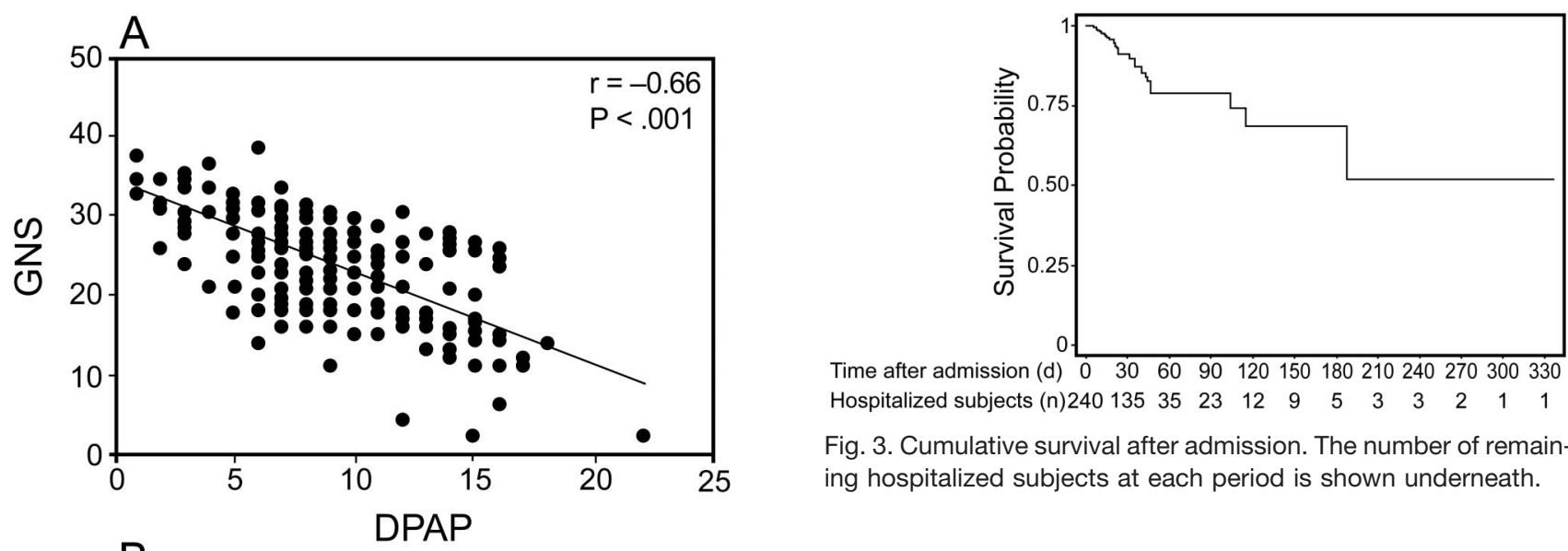

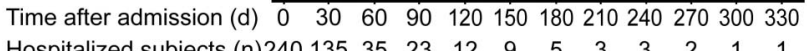

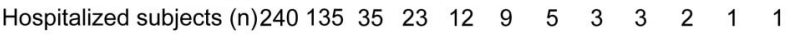

Fig. 3. Cumulative survival after admission. The number of remaining hospitalized subjects at each period is shown underneath.
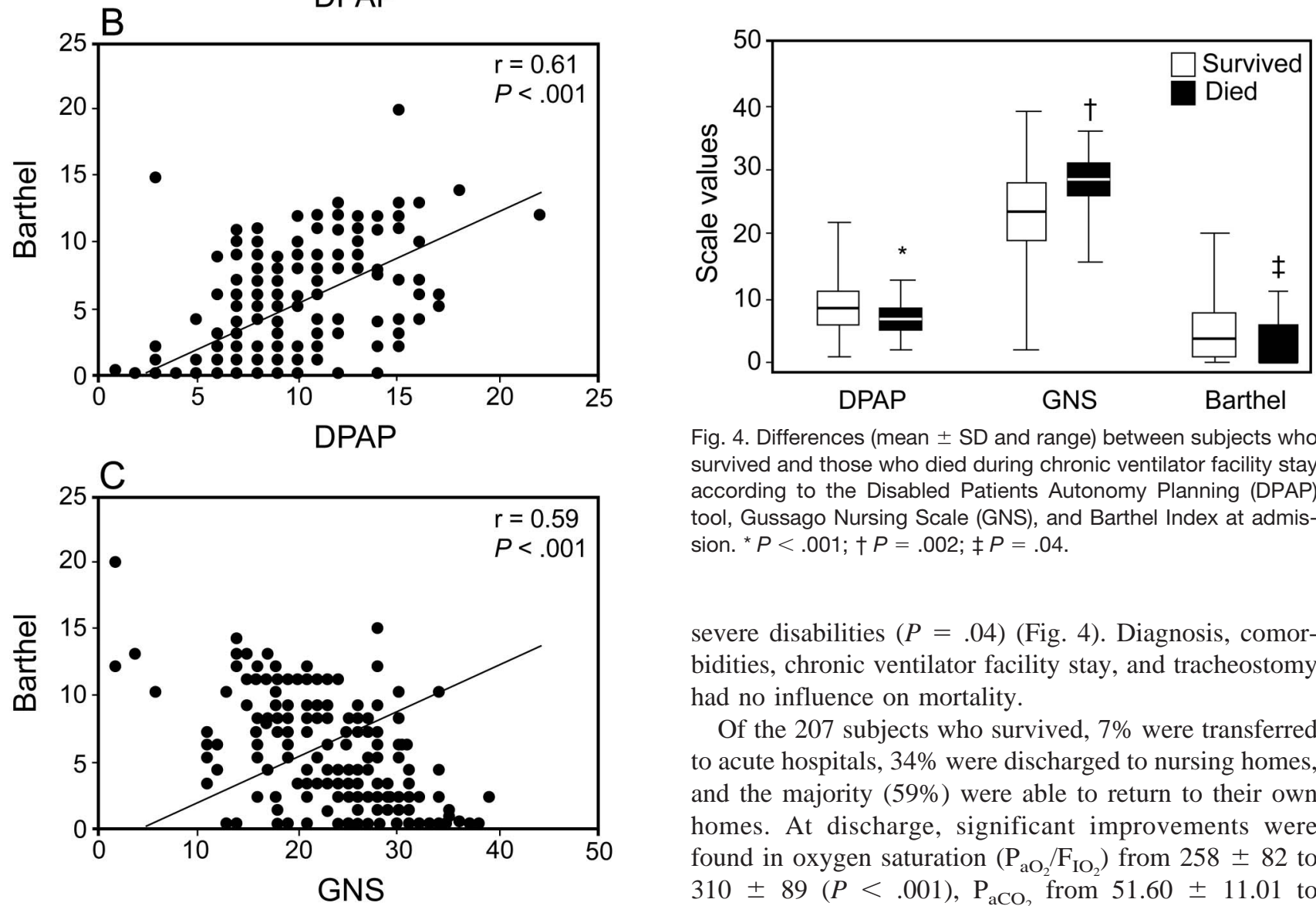

Fig. 4. Differences (mean $\pm S D$ and range) between subjects who survived and those who died during chronic ventilator facility stay according to the Disabled Patients Autonomy Planning (DPAP) tool, Gussago Nursing Scale (GNS), and Barthel Index at admission. ${ }^{*} P<.001 ; \dagger P=.002 ; \ddagger P=.04$.

severe disabilities $(P=.04)$ (Fig. 4). Diagnosis, comorbidities, chronic ventilator facility stay, and tracheostomy had no influence on mortality.

Of the 207 subjects who survived, $7 \%$ were transferred to acute hospitals, $34 \%$ were discharged to nursing homes, and the majority (59\%) were able to return to their own homes. At discharge, significant improvements were found in oxygen saturation $\left(\mathrm{P}_{\mathrm{aO}_{2}} / \mathrm{F}_{\mathrm{IO}_{2}}\right)$ from $258 \pm 82$ to $310 \pm 89(P<.001), \mathrm{P}_{\mathrm{aCO}_{2}}$ from $51.60 \pm 11.01$ to $47.30 \pm 12.32 \mathrm{~mm} \mathrm{Hg}(P=.006)$, and Borg dyspnea from $5.26 \pm 3.03$ to $2.79 \pm 2.90(P<.001)$.

At admission, 165 (68.7\%) subjects were ventilated. Of these, 31 subjects died during the hospital stay, and at discharge, 63 of the 134 survivors $(47 \%)$ were off mechanical ventilation $(P<.001)$. Overall weaning success differed significantly $(P=.007)$ among the different diagnoses $(47.8 \%, 71 \%, 31 \%$, and $48 \%$ for post-surgical subjects and subjects with ARF, COPD, and neurological disease, respectively). Eighteen subjects admitted to the hospital with spontaneous breathing were put on mechanical ventilation during the hospital stay. Subjects were 


\section{Multidisciplinary Care in Acute Respiratory Failure}

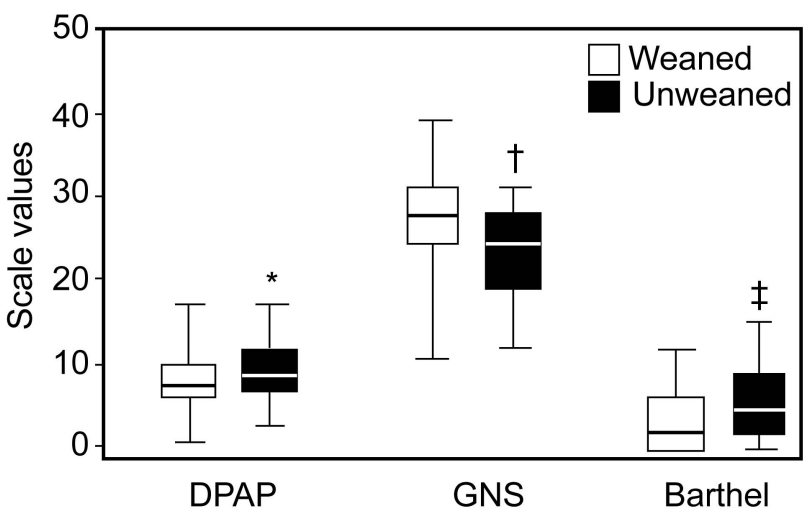

Fig. 5. Differences (mean $\pm S D$ and range) between weaned and unweaned subjects according to the Disabled Patients Autonomy Planning (DPAP) tool, Gussago Nursing Scale (GNS), and Barthel Index at admission. * non-significant $(P=.05)$; $† P<.001$; $\ddagger P=.001$.

weaned in a median time of $10 \mathrm{~d}$ (range of 3-92 d); no significant differences were found in median days for the different diagnoses $(10 \mathrm{~d}$ for post-surgical subjects and subjects with COPD and neurological disease and $12 \mathrm{~d}$ for subjects with ARF).

Compared with unweaned subjects, weaned subjects had higher nursing needs $(P<.001)$ and disability $(P=.001)$ at admission (Fig. 5). At the same time, in the course of their hospital stay, weaned subjects showed a greater improvement in disability $(6.88 \pm 5.47$ vs $3.48 \pm 2.63$, $P<.001)$, autonomy $(9.36 \pm 5.54$ vs $4.79 \pm 4.01$, $P<.001)$, and nursing needs $(15.25 \pm 9.02$ vs $7.26 \pm 5.44$, $P<.001)$. The baseline level of autonomy, hospital stay, tracheostomy, diagnosis, age, malnutrition risk, and gender did not influence weaning success. Of the 183 tracheostomized subjects, 23 died during the hospital stay (all were on mechanical ventilation), whereas at discharge, 52 of the 162 survivors (32\%) were without tracheostomy $(P<.001)$. Compared with subjects eligible for home discharge $(n=122)$, subjects discharged to a nursing home $(n=70)$ presented more frequent neurological diseases (39\% vs $18 \%, P=.01$ ), were more disabled (Barthel Index score of $7.2 \pm 0.51$ vs $12 \pm 0.48, P<.001$ ), were less autonomous (DPAP score of $13 \pm 0.63$ vs $20 \pm 2$, $P<.003$ ), and had more nursing needs (GNS score of $18 \pm 0.8$ vs $10 \pm 0.60, P<.001)$. Mechanical ventilation use and tracheostomy increased the risk of discharge to a nursing home (OR 1.84, $P=.044$; OR 2.47, $P=.003$, respectively).

\section{Discussion}

We have shown that a multidisciplinary care program contributes to reduced disability and nursing needs and to improved autonomy in subjects recovering from ARF in a chronic ventilator facility irrespective of diagnosis; the outcomes for the 3 indices are inter-related. We also showed that (1) mortality is related to ventilation necessity, gender, malnutrition, nursing needs, and autonomy; (2) weaning success differs according to diagnosis, and weaned subjects have more severe disabilities and greater nursing needs at admission but show a greater improvement during the chronic ventilator facility stay; (3) oxygen saturation and dyspnea improve during the stay; and (4) subjects discharged to a nursing home are more disabled and less autonomous, needing more nursing care, home mechanical ventilation, and tracheostomy. Post-discharge care of PMV patients from ICUs often requires their transfer to a chronic ventilator facility, where research has shown that the majority of patients can benefit from an individually tailored rehabilitation program. ${ }^{16-22}$ Most studies, including ours, have focused on mixed diseases, but 3 were performed on subjects with COPD alone. ${ }^{16-18}$ In this severely ill subject subgroup, rehabilitation programs, physiotherapy, and inspiratory muscle training have already been shown to be safe ${ }^{20-22}$ and capable of improving dyspnea, ${ }^{16,17}$ oxygen saturation, ${ }^{18}$ respiratory muscle strength, ${ }^{16,17,19}$ upper and lower limb muscle strength, ${ }^{17-19}$ exercise tolerance, ${ }^{16,17,19,21,22}$ and activities of daily life functional scores, ${ }^{14,20,21}$ and of decreasing hospital stay ${ }^{16,18}$ and weaning time..$^{20}$ Clini et $\mathrm{al}^{21}$ showed that most PMV subjects can benefit from a comprehensive rehabilitation program, suggesting that the degree of improvement of functional disability is associated with survival and weaning success rates. Using the Functional Independence Measure scale as a good indicator of neuromotor disability improvement, Montagnani et al ${ }^{23}$ confirmed the positive effects of rehabilitation on functional status in subjects for whom weaning from mechanical ventilation is difficult.

In our study, the disability, autonomy, and nursing needs improved across all diagnoses, and this finding supports the notion of caring for PMV patients irrespective of their diagnosis. As expected, subjects with neurological disease were more disabled and had more nursing needs, but at the same time, they were able to improve their baseline motor condition by $>190 \%$, autonomy by $72 \%$, and nursing needs by $26 \%$. It is interesting to note that some subjects who were not disabled according to general disability as measured by the DPAP tool were severely motor-disabled according to the Barthel Index. A possible explanation is probably related to the fact that these subjects improved in general autonomy, whereas motor disability remained stable. The 3 different perspectives of patient dependence examined with the DPAP tool (motor disability, comprehensive clinical dependence, and nursing needs) were closely related, confirming that these patients need to be considered as complex regardless of the primary disease that created their disability. We addressed this complexity by using the DPAP triage to orient the health staff in their 


\section{Multidisciplinary Care in Acute Respiratory Failure}

daily care decisions. The DPAP score is an attempt to show a new autonomy/disability value specifically tailored to the needs of this subgroup of patients compared with the accepted standards of the Barthel Index for general disability and the GNS for nursing care.

Lieberman et $\mathrm{al}^{32}$ reported that advanced age and poor functional status as assessed with the Functional Independence Measure are negative factors in the decision to hospitalize a patient in an ICU. In contrast, in our study, although weaned subjects showed higher nursing needs and disabilities at admission, they demonstrated a greater improvement over the course of time, suggesting a positive relation between weaning success and rehabilitation success.

The total weaning success in our subject sample was $47 \%$, with important differences observed among the different diagnoses (ARF and COPD being the best and worst subgroups, respectively). This result seems surprising, but it clearly reflects the death rate: patients with ARF have a high mortality risk, but if they survive, they are easily weanable. In contrast, patients with COPD admitted to ICUs have a lower risk of death but are severely dependent on the ventilator due to their functional impairment after recurrent hospitalizations and use of noninvasive mechanical ventilation. The median time for weaning was $10 \mathrm{~d}$ irrespective of diagnosis, demonstrating that general overall disability is more important than specific disease-related functional impairments in weaning PMV patients. Compared with the previous literature, the lower weaning success rate in our study is probably related to the heterogeneity of our study population and to the specific PMV nature of our subjects. ${ }^{9}$

We have confirmed that patients in a protected environment present a low mortality risk with a weaning rate of $<50 \%$. We have also shown that tracheostomy and stay in a chronic ventilator facility do not affect mortality, weaning, or their timing. Of note, we observed a huge range in the death rate among the different diagnoses, with $23 \mathrm{~d}$ being the median time to death. This reflects the fact that chronic ventilator facilities are requested by ICUs for different reasons: in some cases, to provide an early postacute rehabilitation opportunity for hypoxemic/post-surgical patients with a high risk of death and, in other cases, to provide a later rehabilitation opportunity for severely disabled neurological patients who have survived the ICU. The role in predicting death that gender, need for mechanical ventilation, risk of malnutrition, nursing needs, and low-level global autonomy irrespective of motor disability and diagnosis play is not surprising.

The improvement in oxygen saturation and dyspnea found in our study is in agreement with expected rehabilitation outcomes, as was the long period of stay in our unit. As expected, subjects discharged to a nursing home were more disabled, with lower weaning success, less auton- omy, and more nursing needs (such as mechanical ventilation and tracheostomy). This great burden does not allow families to take direct care of their patient. This fact highlights important ethical and economic considerations that need to be taken into account by decision makers regarding patients who, at discharge from intensive units, require multiple transitions of care, resulting in substantial healthcare costs and persistent profound disability. ${ }^{33}$

\section{Limitations of This Study}

This is a prospective cohort study without a control group, reducing the overgeneralization of findings, since it would have been difficult to separate the effects of a comprehensive rehabilitation program from spontaneous healing and functional recovery. In any case, lack of a rehabilitation program in this particular subgroup of subjects (ie, control group) would have been unethical and unacceptable according to our local ethics committee. The variety of diagnoses may reduce the generalizability of measured outcomes. In contrast, the heterogeneity of our subject population mirrors the real-life setting of chronic illnesses that require intensive care.

\section{Conclusions}

This study emphasizes that specialized rehabilitation dedicated to these complex patients based on an individually tailored approach and continuity of care over time should be an integral part of their multidisciplinary treatment. In patients discharged from ICUs and irrespective of their diagnoses, a multidisciplinary care program contributes to reduced disability and nursing needs and improved autonomy, with these indices being inter-related. Mortality and weaning are related to clinical and disability status and to their reciprocal improvement over time. Different diagnoses have different rates of weaning success. Patients discharged to a nursing home are the most severely disabled.

\section{ACKNOWLEDGMENTS}

We thank Ms Rosemary Allpress for English revision of this manuscript.

\section{REFERENCES}

1. Vitacca M, Nava S. Incomplete network for survivors of catastrophic illness after release from ICUs. Respir Care 2013;58(2):383-385.

2. Topp R, Ditmyer M, King K, Doherty K, Hornyak J 3rd. The effect of bed rest and potential of rehabilitation on patients in the intensive care unit. AACN Clin Issues 2002;13(2):263-276.

3. Nava S, Vitacca M. Chronic ventilator facilities, 2nd edition. In: Tobin MJ, editor. Principles and practice of mechanical ventilation. New York: McGraw-Hill; 2006:691.

4. Sacanella E, Pérez-Castejón JM, Nicolás JM, Masanés F, Navarro M, Castro P, López-Soto A. Mortality in healthy elderly patients after ICU admission. Intensive Care Med 2009;35(3):550-555. 


\section{Multidisciplinary Care in Acute Respiratory Failure}

5. Stoller JK, Xu M, Mascha E, Rice R. Long-term outcomes for patients discharged from a long-term hospital-based weaning unit. Chest 2003;124(5):1892-1899.

6. Schönhofer B, Euteneuer S, Nava S, Suchi S, Köhler D. Survival of mechanically ventilated patients admitted to a specialised weaning centre. Intensive Care Med 2002;28(7):908-916.

7. Engoren M, Arslanian-Engoren C, Fenn-Buderer N. Hospital and long-term outcome after tracheostomy for respiratory failure. Chest 2004;125(1):220-227.

8. Cox CE, Carson SS, Govert JA, Chelluri L, Sanders GD. An economic evaluation of prolonged mechanical ventilation. Crit Care Med 2007;35(8):1918-1927.

9. Boles JM, Bion J, Connors A, Herridge M, Marsh B, Melot C, et al. Weaning from mechanical ventilation. Eur Respir J 2007;29(5): 10331056.

10. Corrado A, Roussos C, Ambrosino N, Confalonieri M, Cuvelier A, Elliott M, et al. Respiratory intermediate care units: a European survey. Eur Respir J 2002;20(5):1343-1350.

11. Byrick RJ, Mazer CD, Caskennette GM. Closure of an intermediate care unit. Impact on critical care utilization. Chest 1993;104(3):876-881.

12. Robson V, Poynter J, Lawler PG, Baudouin SV. The need for a regional weaning centre, a one-year survey of intensive care weaning delay in the Northern Region of England. Anaesthesia 2003;58(2):161-165.

13. Clini E, Ambrosino N. Early physiotherapy in the respiratory intensive care unit. Respir Med 2005;99(9):1096-1104.

14. Thomsen GE, Snow GL, Rodriguez L, Hopkins RO. Patients with respiratory failure increase ambulation after transfer to an intensive care unit where early activity is a priority. Crit Care Med 2008;36(4): 1119-1124.

15. Gosselink R, Bott J, Johnson M, Dean E, Nava S, Norrenberg M, et al. Physiotherapy for adult patients with critical illness: recommendations of the European Respiratory Society and European Society of Intensive Care Medicine Task Force on Physiotherapy for Critically Ill Patients. Intensive Care Med 2008;34(7):1188-1199.

16. Nava S. Rehabilitation of patients admitted to a respiratory intensive care unit. Arch Phys Med Rehabil 1998;79(7):849-854.

17. Porta R, Vitacca M, Gilè LS, Clini E, Bianchi L, Zanotti E, Ambrosino N. Supported arm training in patients recently weaned from mechanical ventilation. Chest 2005;128(4):2511-2520.

18. Zanotti E, Felicetti G, Maini M, Fracchia C. Peripheral muscle strength training in bed-bound patients with COPD receiving mechanical ventilation. Effect of electrical stimulation. Chest 2003;124(1):292-296.

19. Chiang LL, Wang LY, Wu CP, Wu HD, Wu YT. Effects of physical training on functional status in patients with prolonged mechanical ventilation. Phys Ther 2006;86(9):1271-1281.

20. Martin UJ, Hincapie L, Nimchuk M, Gaughan J, Criner GJ. Impact of whole-body rehabilitation in patients receiving chronic mechanical ventilation. Crit Care Med 2005;33(10):2259-2265.
21. Clini EM, Crisafulli E, Antoni FD, Beneventi C, Trianni L, Costi S, et al. Functional recovery following physical training in tracheotomized and chronically ventilated patients. Respir Care 2011;56(3): 306-313.

22. Bailey P, Thomsen GE, Spuhler VJ, Blair R, Jewkes J, Bezdjian L, et al. Early activity is feasible and safe in respiratory failure patients. Crit Care Med 2007;35(1):139-145.

23. Montagnani G, Vagheggini G, Panait Vlad E, Berrighi D, Pantani L, Ambrosino N. Use of the functional independence measure following a weaning program from mechanical ventilation in difficult to wean patients. Phys Ther 2011;91(7):1109-1115.

24. Polverino E, Nava S, Ferrer M, Ceriana P, Clini E, Spada E, et al. Patients' characterization, hospital course and clinical outcomes in five Italian respiratory intensive care units. Intensive Care Med 2010; 36(1):137-142.

25. Vitacca M, Vianello A, Colombo D, Clini E, Porta R, Bianchi L, et al. Comparison of two methods for weaning patients with chronic obstructive pulmonary disease requiring mechanical ventilation for more than 15 days. Am J Respir Crit Care Med 2001;164(2):225230.

26. Parmelee PA, Thuras PD, Katz IR, Lawton MP. Validation of the Cumulative Illness Rating Scale in a geriatric residential population. J Am Geriatr Soc 1995;43(2):130-137.

27. Borg GA. Psychophysical bases of perceived exertion. Med Sci Sports Exerc 1982;14(5):377-381.

28. The 'MUST' explanatory booklet. A guide to the 'Malnutrition Universal Screening Tool' ('MUST') for adults. In: Todorovic V, Russell C, and Elia M, editors. Worcs, United Kingdom: British Association for Parenteral and Enteral Nutrition; 2011.

29. Knaus WA, Zimmerman JE, Wagner DP, Draper EA, Lawrence DE. APACHE-acute physiology and chronic health evaluation: a physiologically based classification system. Crit Care Med 1981;9(8):591597.

30. Mahoney FI, Barthel DW. Functional evaluation: the Barthel Index. Md State Med J 1965;14:61-65.

31. Clini E, Vitacca M, Ambrosino N. Dependence Nursing Scale: a new method to assess the effect of nursing workload in respiratory intermediate intensive care unit. Respir Care 1999;44(1):29-37.

32. Lieberman D, Nachshon L, Miloslavsky O, Dvorkin V, Shimoni A, Zelinger $\mathrm{J}$, et al. Elderly patients undergoing mechanical ventilation in and out of intensive care units: a comparative, prospective study of 579 ventilations. Crit Care 2010;14(2):R48.

33. Unroe M, Kahn JM, Carson SS, Govert JA, Martinu T, Sathy SJ, et al. One-year trajectories of care and resource utilization for recipients of prolonged mechanical ventilation. Ann Intern Med 2010; 153(3):167-175. 